\title{
Factors Affecting Nurses' Job Satisfaction in Rural and Urban Acute Care Settings:
}

\section{A PRISMA Systematic Review}

This review aims to systemically describe the findings of primary studies in order to identify the intrinsic and extrinsic factors that affect nurses' job satisfaction using PRISMA guidelines. It also aims to analyze the finding according to the two-factor theory; and compare studies based on rural and urban settings.

Two reviewers completed study selection, screening, and quality assessment. After data extraction, content analysis was used to categorize identified factors into themes.

Thirty-eight studies were selected for this review. Extrinsic factors reported in the findings were: work conditions $(n=17)$, monetary benefits $(n=5)$, hospital policies $(n=6)$, supervision $(n=7)$, interpersonal relationships $(n=8)$, organization culture and emotional display norms in the organization $(n=2)$, job security $(n=1)$, and professional status $(n=2)$. Intrinsic factors reported in review were responsibility $(n=9)$, growth and advancement $(n=7)$, psychological demands $(n=2)$, recognition $(n=2)$, and job achievement $(n=1)$. Furthermore, personal factors were classified into demographic variables, and behavioral/emotional factors and each of them reported in seven studies. Two studies reported community factors. Twenty-two studies reported the hospital location. Urban studies focused on extrinsic factors while there was more balance in rural studies.

There have been many published studies discuss the factors associated with nurses' job satisfaction. However, more studies are needed to examine the impact of intrinsic and extrinsic factors on nurses' job satisfaction using more robust research methodology especially in rural and urban context. The two-factor theory can be used to provide conceptual clarity regarding the impact of intrinsic and extrinsic factors on job satisfaction. 